\title{
Intención emprendedora en estudiantes universitarios de Ingeniería Industrial Administrativa de la Universidad Católica Santa María la Antigua (USMA).
}

\author{
Armando José Espino Cruz ${ }^{1}$ y Samuel Vásquez González ${ }^{2, *}$ \\ ${ }^{1}$ Psicólogo, Fundación para el Desarrollo Integrado Sustentable (FUDIS), Panamá, República de Panamá. \\ 2 Profesor, Facultad de Ingeniería y Tecnología, Universidad Católica Santa María la Antigua (USMA), \\ Panamá, República de Panamá. \\ *Autor para correspondencia. Email: svasquezg@usma.com.pa
}

Recibido: 2 de diciembre de 2015

Aceptado: 10 de diciembre de 2015

\begin{abstract}
Addressing the motives that drive individuals to opt for entrepreneurship is relevant because we can shed lights on the process of how a person becomes a successful entrepreneur. This knowledge is applicable to entrepreneur's formation, a task in which students require help in aspects such as: perceived convenience, perceived viability, whether the cultural values and beliefs promote entrepreneurship as a lifestyle. This study offers a perspective upon entrepreneurship intention in students enrolled in the first and the fourth year of Industrial Engineering at Universidad Católica Santa María la Antigua (USMA). Our aim was to assess entrepreneurship intention and perceptions about factors that contribute toward entrepreneurship, in order to explore if these are related to the year enrolled. Participants were tested with a questionnaire that included the Entrepreneurial Self-Efficacy Scale, and information from the Global Entrepreneurship Monitor. Results show that fourth-year students have a higher level of entrepreneurship self-efficacy, while first-year students have better attitudes towards entrepreneurship.

Keywords: GEM Model; Self-Efficacy; Entrepreneurship; Global Entrepreneurship Monitor; Entrepreneur Capacity.

\section{Resumen}

Abordar los motivos que tienen los individuos para optar por el emprendimiento es relevante, ya que se puede ofrecer una aproximación que permita entender cómo se convierte uno en un emprendedor y, más aun, en un emprendedor exitoso. Este conocimiento es aplicable a la formación de emprendedores, tarea para la cual debe ayudarse a los estudiantes a responder cuestiones tales como: el grado en que se siente atraído para convertirse en empresario (conveniencia percibida), el grado en que se
\end{abstract}


Invest. pens. crit.

Vol. 3, No. 3, septiembre-diciembre 2015

pp. $13-26$

consideran personalmente capaz para ser empresarios (viabilidad percibida), si los valores o creencias que conforman la cultura incentivan o no el emprendimiento como opción de vida. El presente estudio ofrece una perspectiva sobre la intención emprendedora en estudiantes de primer ingreso y alumnos graduandos de Ingeniería Industrial Administrativa de la Universidad Católica Santa María la Antigua (USMA). El objetivo es evaluar la intención emprendedora y la percepción emprendedora sobre algunos elementos que inciden en la intención emprendedora de estos estudiantes, con el fin de explorar diferencias entre los grupos. Se aplicó un instrumento construido con base en la escala de autoeficacia y la encuesta del Global Entrepreneurship Monitor. Los resultados demuestran que los estudiantes de cuarto año tienen un mayor nivel de autoeficacia emprendedora, mientras que los de primero muestran mejores actitudes hacia el emprendimiento.

Palabras clave: Modelo GEM; Autoeficacia; Emprendimiento; Global Entrepreneurship Monitor; Capacidad Emprendedora.

\section{Introducción}

El individuo, como figura protagónica del emprendimiento, pone en marcha la actitud y capacidad emprendedora para crear empresas, un proyecto o un sistema de vida. A partir de una conceptualización de oportunidades-que percibe en el entorno-las traduce en nuevas soluciones, productos y servicios que cubren las necesidades del mercado de una forma más eficiente.

Actualmente, la investigación en torno al emprendedor se centra menos en los rasgos y más en los comportamientos; es decir, en torno a la verdadera forma de actuar del emprendedor: qué hace, qué sabe hacer o qué debe saber hacer. Un factor fundamental en esta línea es la competencia empresarial, la cual difiere entre emprendedores. Esta competencia se forma a través del empleo de capacidades o habilidades empresariales, las cuales pueden ser entrenadas.

Cuando uno se sumerge en el discurso académico sobre el emprendimiento, es inevitable encontrarse con la tan nombrada cuestión sobre si los emprendedores nacen o se forman.

Abordar los motivos que tienen los individuos para optar por el emprendimiento es relevante, ya que se puede ofrecer una aproximación que permita entender cómo se convierte uno en un emprendedor y, más aun, en un emprendedor exitoso; que se puede, desde la universidad, cambiar la intención de los estudiante hacia el emprendimiento.

Para esto, se debe ayudar a los estudiantes a responder cuestiones tales como: el grado en que se siente atraído para convertirse en empresario (conveniencia percibida), el grado en que se consideran personalmente capaces para ser empresarios (viabilidad percibida), si los valores o creencias que conforman la cultura incentivan o no el emprendimiento como opción de vida (Shapero y Sokol, 1982).

Considerando los trabajos de diferentes autores sobre la intención emprendedora (Elfving, Brännback y Carsud, 2009; Moriano, Palací y Morales, 2006) y los rasgos comunes que aparecen en estos trabajos, 
hemos desarrollado un modelo que se basa en uno de los principales rasgos de personalidad que emerge estudiar la intención emprendedora: la autoeficacia.

Sin embargo, un marco de estudio de la intención emprendedora debe incluir variables como motivación, valoración de oportunidades, el rol de las normas sociales y culturales, todos evasivos en el modelo de intención emprendedora. De lo contrario es limitante el análisis de la intención emprendedora. Por lo tanto, el presente estudio toma como referencia el Modelo del Global Entrepreneurship Monitor (GEM), que evalúa actitudes emprendedoras que son importantes porque expresan los sentimientos generales de la población hacia los emprendedores y el emprendimiento.

Por otro lado, la autoeficacia no impacta en la formación de intención emprendedora de manera aislada o directa. De allí la importancia de incluir el análisis de otros factores en un modelo de intención emprendedora. La motivación, por ejemplo, determinará las metas de las personas en la vida. En combinación con la autoeficacia, la motivación impacta la opción de carrera en la vida y la intención de emprender.

La autoeficacia, según Bandura (1977), impacta a su vez en un mayor compromiso, lo que hace que la persona se sienta más confortable a continuar su opción de vida. De allí que el presente estudio incluye un rasgo de personalidad común en modelos de intención emprendedora y factores de actitudes hacia el emprendimiento, como la motivación, percepción de oportunidades, así como la cultura y el rol social.

Este estudio se centra en aplicación de la Escala de Autoeficacia Emprendedora (De Noble, Dong y Sanford, 1999) en estudiantes de primer ingreso y último año de la carrera de Ingeniería Industrial Administrativa para evaluar las creencias de los estudiantes sobre su propia capacidad para crear y gestionar una nueva empresa con éxito. Al final buscamos identificar si existen diferencias significativas entre ambos grupos.

\section{Materiales y métodos}

Se realizó una investigación de tipo observacional, utilizando un diseño transversal, con un alcance correlacional.

\section{$\underline{\text { Participantes }}$}

Participaron 48 estudiantes de la carrera de Ingeniería Industrial Administrativa de la Universidad Católica Santa María La Antigua de Panamá. 24 eran estudiantes de primer ingreso (primer año de la licenciatura) y los otros 24 eran estudiantes graduandos (cuarto año de la licenciatura). Todos los participantes estaban matriculados en el turno diurno de la carrera. 
Invest. pens. crit.

Vol. 3, No. 3, septiembre-diciembre 2015

pp. 13-26

\section{$\underline{\text { Medidas }}$}

El cuestionario de evaluación, que llamamos Encuesta de Intención Emprendedora en Estudiantes Universitarios, fue diseñado de manera sencilla, con términos relativamente fáciles de entender, y todas las preguntas con respuestas de opciones múltiples. El cuestionario inclúa las siguientes medidas:

a) Preguntas generales: En esta sección del cuestionario se incluyen preguntas de carácter personal del estudiante y sus padres, como su edad, ingreso familiar y algunos aspectos de su situación laboral. Además, se incluye una pregunta clave para este estudio, la pregunta 1.8, que tiene que ver con su opción de vida futura.

b) Escala de Autoeficacia Emprendedora (Moriano, Palací y Morales, 2006). Se trata de la adaptación española de la escala Entrepreneurial Self-Efficacy (ESE; De Noble, Dong y Sanford, 1999). Está compuesta por 23 ítems que evalúan seis dimensiones que recogían las principales tareas que debe desarrollar un emprendedor para crear con éxito su propia empresa, a saber: 1 . Desarrollar nuevos productos y oportunidades de mercado; 2. Construir un entorno innovador; 3. Iniciar relaciones con inversores; 4. Definir el objetivo central del negocio; 5. Afrontar cambios inesperados; y 6. Desarrollar los recursos humanos clave.

Cada reactivo presenta opciones de respuestas en formato tipo Likert de 5 puntos, desde "completamente incapaz" (1) a "completamente capaz" (5). Los valores asignados a los ítems son sumados (o promediados) para obtener una única puntuación global en auto-eficacia emprendedora. También se puede calcular la puntuación específica del sujeto para cada una de las seis dimensiones.

c) Percepción ante aseveraciones relacionadas con Ser Emprendedor: Las últimas tres secciones del cuestionario están relacionadas con la percepción que tenga el estudiante ante una serie de aseveraciones sobre la Motivación para Emprender (sección 3), sobre las Normas Sociales y Culturales (sección 4) y sobre las Oportunidades para Emprender (sección 5).

Cada una de esas aseveraciones el estudiante debía responder si, según su opinión, esa aseveración era Completamente falsa, Más bien falsa, Ni cierto ni falso, Más bien Cierto o Completamente cierto.

\section{Procedimiento.}

Una vez recolectados los datos a través de la aplicación la Encuesta a ambos grupos de estudiantes, se procedió a la transcripción de los datos, diseñando las tablas y gráficas respectivas, que servirían de base para la preparación del análisis de los datos recopilados, que servirían de base para la fase final del estudio. 


\section{Resultados}

\section{Autoeficacia Emprendedora}

Dimensión 1: Descubrir nuevos productos y oportunidades de mercado. En relación al reconocimiento de nuevas oportunidades en el mercado para nuevos productos y servicios (2.3), el 100\% de los graduandos se siente capaces de completar la tarea en algún grado mayor o menor, mientras que del grupo de Primer Ingreso, sólo el 96\% se siente capaz.

Respecto al descubrimiento de nuevas formas para mejorar los productos existentes (2.6), el 88\% de los estudiantes I Año se creen capaces de completar la tarea en mayor o menor grado, en relación al $100 \%$ de los graduandos.

Referente a la tarea de Identificar nuevas áreas de crecimiento potencial (2.8), el 100\% del grupo de IV Año creen ser capaces en mayor o menor grado, en relación al 92\% de los de primer ingreso.

A la tarea de diseñar productos que resuelvan problemas corrientes (2.12), 91\% de los graduandos piensan que son capaces de completar la tarea en mayor o menor grado, con respecto al $83 \%$ de los de I Año.

El ítem relacionado a crear productos que satisfagan las necesidades no cubiertas de los clientes (2.16), $100 \%$ de los graduandos se creen capaces en mayor o menor grado, mientras que sólo el 83\% de los de I Año lo creen.

Sobre el tema de formular unas acciones rápidas para perseguir las oportunidades (2.17), 100\% de los graduandos y de I Año se creen capaces en mayor o menor grado.

Tabla 1.

Dimensión 1: Descubrir nuevos productos y oportunidades de mercado

\begin{tabular}{cccc}
\hline Ítem & Grupo de estudio & Incapaz & Capaz \\
\hline 2.3. & I año & $4 \%$ & $96 \%$ \\
& IV año & $0 \%$ & $100 \%$ \\
2.6. & I año & $13 \%$ & $88 \%$ \\
& IV año & $0 \%$ & $100 \%$ \\
2.8. & I año & $8 \%$ & $92 \%$ \\
& IV año & $0 \%$ & $100 \%$ \\
2.12. & I año & $17 \%$ & $83 \%$ \\
& IV año & $13 \%$ & $91 \%$ \\
2.16. & I año & $17 \%$ & $83 \%$ \\
& IV año & $0 \%$ & $100 \%$ \\
2.17. & I año & $0 \%$ & $100 \%$ \\
& IV año & $0 \%$ & $100 \%$ \\
2.19. & I año & $17 \%$ & $83 \%$ \\
& IV año & $8 \%$ & $92 \%$ \\
\hline Promedio & I año & $\mathbf{1 1} \%$ & $\mathbf{8 9} \%$ \\
& IV año & $\mathbf{2 \%}$ & $\mathbf{9 8 \%}$ \\
\hline
\end{tabular}


Invest. pens. crit.

Vol. 3, No. 3, septiembre-diciembre 2015

pp. $13-26$

La utilización de antiguos conceptos comerciales de una nueva manera (2.19), el grupo de IV Año $92 \%$ se creen capaces en mayor o menor grado, en relación al 83\% de I Año.

Como podemos observar en la Tabla 1, los estudiantes de IV Año se sienten 98\% capaces, en mayor o menor grado, de completar la tarea de descubrir nuevos productos y oportunidades de mercado, en relación al 89\% de los de I Año. Sólo 11\% de los estudiantes de I Año se creen incapaces parcial o completamente en relación al 2\% de los de IV Año.

Dimensión 2: Desarrollar los recursos humanos claves. En lo que respecta al ítem trabajar eficazmente bajo un continuo estrés, presión y conflicto (2.1), 96\% de los estudiantes de IV Año se creen con capacidad, en mayor o menor grado, en relación con el $92 \%$ de los de I Año.

El ítem de Reclutar y entrenar a los empleados clave (2.4), 100\% de los estudiantes de IV Año se creen con capacidad, en mayor o menor grado, en relación con el $96 \%$ de los de I Año.

Tabla 2.

\begin{tabular}{cccc} 
Dimensión 2: Desarrollar los recursos bumanos claves \\
\hline Ítem & Grupo de estudio & Incapaz & Capaz \\
\hline 2.1. & I año & $8 \%$ & $92 \%$ \\
& IV año & $4 \%$ & $96 \%$ \\
2.4. & I año & $4 \%$ & $96 \%$ \\
& IV año & $0 \%$ & $100 \%$ \\
2.9. & I año & $17 \%$ & $83 \%$ \\
& IV año & $9 \%$ & $91 \%$ \\
\hline Promedio & I año & $\mathbf{1 0 \%}$ & $\mathbf{9 0} \%$ \\
& IV año & $\mathbf{4} \%$ & $\mathbf{9 6} \%$ \\
\hline
\end{tabular}

En lo que corresponde al ítem 2.9, el 91\% de los estudiantes de IV Año se creen con capacidad, en mayor o menor grado, en relación con el 83\% de los de I Año.

Los estudiantes de IV Año se sienten 96\% capaces en mayor o menor grado de completar la tarea de desarrollar recursos humanos claves, en relación al 90\% de los de I Año.

Sólo 10\% de los de primer año se creen incapaces parcial o Completamente, en relación al 4\% de los de IV Año.

Dimensión 3 - Iniciar relaciones con inversores. El ítem Desarrollar y mantener relaciones favorables con potenciales inversores (2.2), en este caso, el 100\% de ambos grupos de estudio se sienten, en mayor o menor grado, con capacidad de llevar a cabo esta tarea.

El Desarrollar relaciones con personal clave para obtener capital, contrario a los resultados anteriores (2.7), 100\% de los estudiantes de I Año se sienten capaces, en mayor o menor grado, en relación con el 96\% de los de IV Año. 
El ítem Identificar recursos potenciales de financiación (2.13), 92\% de los estudiantes de IV Años se creen con capacidad, en mayor o menor grado, en relación con el 75\% de los de I Año.

Tabla 3.

\begin{tabular}{|c|c|c|c|}
\hline Ítem & Grupo de estudio & Incapaz & Capaz \\
\hline \multirow[t]{2}{*}{2.2.} & I año & $0 \%$ & $100 \%$ \\
\hline & IV año & $0 \%$ & $100 \%$ \\
\hline \multirow[t]{2}{*}{2.7.} & I año & $0 \%$ & $100 \%$ \\
\hline & IV año & $4 \%$ & $96 \%$ \\
\hline \multirow[t]{2}{*}{2.13} & I año & $25 \%$ & $75 \%$ \\
\hline & IV año & $9 \%$ & $91 \%$ \\
\hline Pro & $\begin{array}{c}\text { I año } \\
\text { IV año }\end{array}$ & $\begin{array}{l}8 \% \\
4 \%\end{array}$ & $\begin{array}{l}92 \% \\
96 \%\end{array}$ \\
\hline
\end{tabular}

Los estudiantes de IV Año se sienten 96\% capaces en mayor o menor grado de completar la tarea de iniciar relaciones con inversores, en relación al 92\% de los de I Año. Sólo 8\% de los de I Año se creen incapaces parcial o completamente en relación al 4\% de los de IV Año.

Encontramos un ítem “desarrollar relaciones con personas clave para obtener capital” (2.7) donde el análisis mostró mayor puntuación en estudiantes de I Año en relación al de IV Año.

Dimensión 4: Construir un entorno innovador. El ítem Crear un entorno de trabajo que permita a las personas ser más su propio jefe (2.14), 92\% de ambos grupos nos indican que se creen con capacidad, en mayor o menor grado. Sin embargo, el 4\% de los de IV Año se sienten completamente incapaces, y el $8 \%$ de los de I Año se sienten algo incapaces.

El ítem Desarrollar un entorno laboral que promueva que las personas intenten hacer cosas nuevas (2.18), 100\% de los estudiantes de I Año se creen con capacidad, en mayor o menor grado, en relación con el 96\% de los de IV Año.

Otro hallazgo importante es el que encontramos en este ítem, los estudiantes de I Año se sienten en la totalidad de los casos más capaces que los de IV Año con una diferencia de 4 puntos porcentuales.

Con respecto al tema del ítem 2.21, en ambos grupos, el 96\% de los estudiantes se creen con capacidad, en mayor o menor grado. Lo interesante que el $4 \%$ de los estudiantes del grupo de IV Año se sienten completamente incapaces.

Identificar y construir equipos de gestión (2.22), es una cuestión que el 92\% de los jóvenes de I Año se creen con capacidad, en mayor o menor grado, en relación con el 87\% de los de IV Año.

Otro hallazgo importante se da en este ítem, hay una diferencia de 5 puntos porcentuales a favor de los estudiantes de I Año que se sienten con mayor capacidad que los de IV Año. 
Invest. pens. crit.

Vol. 3, No. 3, septiembre-diciembre 2015

pp. $13-26$

Tabla 4.

Dimensión 4: Construir un entorno innovador.

\begin{tabular}{cccc}
\hline Ítem & Grupo de estudio & Incapaz & Capaz \\
\hline 2.14. & I año & $8 \%$ & $92 \%$ \\
\multirow{2}{*}{2.18.} & IV año & $8 \%$ & $91 \%$ \\
\multirow{2}{*}{2.21.} & I año & $0 \%$ & $100 \%$ \\
\multirow{2}{*}{2.22.} & IV año & $4 \%$ & $96 \%$ \\
& I año & $4 \%$ & $96 \%$ \\
& IV año & $4 \%$ & $96 \%$ \\
& I año & $8 \%$ & $92 \%$ \\
Promedio & IV año & $13 \%$ & $87 \%$ \\
\hline & I año & $\mathbf{5 \%}$ & $\mathbf{9 5 \%}$ \\
& IV año & $\mathbf{7 \%}$ & $\mathbf{9 3} \%$ \\
\hline
\end{tabular}

Los estudiantes de I Año se sienten 95\% capaces, en mayor o menor grado, de completar la tarea de construir un entorno innovador, en relación al 93\% de los de IV Año. Sólo 5\% de los de Primer Ingreso se creen incapaces parcial o completamente en relación al 8\% de los de IV Año.

En esta dimensión encontramos un primer hallazgo interesante de análisis, dado que hay mayor cultura innovadora en los estudiantes de I Año en contraposición con los de IV Año.

Dimensión 5: Afrontar cambios inesperados. Tolerar los cambios inesperados en las condiciones del negocio (2.11), 88\% de los estudiantes de I Año se creen con capacidad, en mayor o menor grado, en relación con el 91\% de los de IV Año.

Con respecto al tema de Persistir frente a la adversidad (2.15), en ambos grupos el 100\% de ellos se creen con capacidad, en mayor o menor grado, de afrontar la adversidad.

El ítem Determinar si el negocio va bien (2.20), 96\% de los estudiantes de I Año se creen con capacidad, en mayor o menor grado, en relación con el 100\% de los de IV Año.

Tabla 5.

Dimensión 5: Afrontar cambios inesperados.

\begin{tabular}{cccc}
\hline Ítem & Grupo de estudio & Incapaz & Capaz \\
\hline 2.11. & I año & $12 \%$ & $88 \%$ \\
& IV año & $9 \%$ & $91 \%$ \\
2.15. & I año & $0 \%$ & $100 \%$ \\
& IV año & $0 \%$ & $100 \%$ \\
2.20. & I año & $4 \%$ & $96 \%$ \\
& IV año & $0 \%$ & $100 \%$ \\
\hline Promedio & I año & $\mathbf{6} \%$ & $\mathbf{9 4} \%$ \\
& IV año & $\mathbf{3} \%$ & $\mathbf{9 7 \%}$ \\
\hline
\end{tabular}


Los estudiantes de I Año se sienten 94\% capaces, en mayor o menor grado, de completar la tarea de construir un entorno innovador en relación al 97\% de los de IV Año. Sólo 6\% de los de Primer Ingreso se creen incapaces parcial o completamente en relación al 3\% de los de IV Año.

Dimensión 6: Definir el objetivo central del negocio. Con respecto al ítem 2.5, el 92\% de los estudiantes de I Año se creen con capacidad, en mayor o menor grado, de realizar esta tarea, en relación con el 96\% de los de IV Año.

El ítem 2.10, en ambos grupos el 92\% de los estudiantes de I Año se creen con capacidad, en mayor o menor grado.

En relación con el ítem 2.23, 87\% de los estudiantes de I Año se creen con capacidad, en mayor o menor grado, en relación con el 91\% de los de IV Año.

Tabla 6.

Dimensión 6: Definir el objetivo central del negocio

\begin{tabular}{cccc}
\hline Ítem & Grupo de estudio & Incapaz & Capaz \\
\hline 2.5. & I año & $8 \%$ & $92 \%$ \\
& IV año & $4 \%$ & $96 \%$ \\
2.10. & I año & $8 \%$ & $92 \%$ \\
\multirow{2}{*}{2.23.} & IV año & $8 \%$ & $92 \%$ \\
& I año & $13 \%$ & $87 \%$ \\
& IV año & $9 \%$ & $91 \%$ \\
\hline Promedio & I año & $\mathbf{1 0 \%}$ & $\mathbf{9 0} \%$ \\
& IV año & $\mathbf{7 0} \%$ & $\mathbf{9 3} \%$ \\
\hline
\end{tabular}

Los estudiantes de I Año se sienten 90\% capaces en mayor o menor grado de completar la tarea de construir un entorno innovador en relación al 93\% de los de IV Año. Sólo 10\% de los de I Año se creen incapaces parcial o completamente, en relación al 7\% de los de IV Año.

Resumen de análisis de escala de Autoeficacia Emprendedora

La evaluación de la escala permite medir las creencias o el sentir de los estudiantes sobre su propia capacidad para crear y gestionar una nueva empresa con éxito. En este estudio se buscó establecer si existen diferencias significativas entre un grupo de I Año, primer ingreso, respecto a un grupo IV Año, graduandos.

En general, se muestra que de I Año el 92\% de los estudiantes se creen capaces de crear y gestionar una empresa con éxito en relación a 95\% de IV Año, por lo que concluimos que no existe mayor diferencia significativa entre ambos grupos y, como se esperaba, el grupo de IV Año obtuvo mayor puntuación en la escala de Autoeficacia Emprendedora.

Tabla 
Invest. pens. crit.

Vol. 3, No. 3, septiembre-diciembre 2015

pp. 13-26

\begin{tabular}{|c|c|c|}
\hline Grupo de estudio & Incapaz & Capaz \\
\hline \multicolumn{3}{|c|}{ Dimensión 1: Descubrir nuevos productos y oportunidades de mercado } \\
\hline I año & $11 \%$ & $89 \%$ \\
\hline IV año & $2 \%$ & $98 \%$ \\
\hline \multicolumn{3}{|c|}{ Dimensión 2: Desarrollar los Recursos Humanos Claves } \\
\hline I año & $10 \%$ & $90 \%$ \\
\hline IV año & $4 \%$ & $96 \%$ \\
\hline \multicolumn{3}{|c|}{ Dimensión 3: Iniciar relaciones con inversores } \\
\hline I año & $8 \%$ & $92 \%$ \\
\hline IV año & $4 \%$ & $96 \%$ \\
\hline \multicolumn{3}{|c|}{ Dimensión 4: Construir un entorno innovador } \\
\hline I año & $5 \%$ & $95 \%$ \\
\hline IV año & $7 \%$ & $93 \%$ \\
\hline \multicolumn{3}{|c|}{ Dimensión 5: Afrontar cambios inesperados } \\
\hline I año & $6 \%$ & $94 \%$ \\
\hline IV año & $3 \%$ & $97 \%$ \\
\hline \multicolumn{3}{|c|}{ Dimensión 6: Definir el objetivo central del negocio } \\
\hline I año & $10 \%$ & $90 \%$ \\
\hline IV año & $7 \%$ & $91 \%$ \\
\hline \multirow[t]{2}{*}{ Promedio } & $8 \%$ & $92 \%$ \\
\hline & $5 \%$ & $95 \%$ \\
\hline
\end{tabular}

Sí llamó la atención, como se señaló anteriormente, que hay mayor Cultura Innovadora en los estudiantes de I Año en contraposición con los de IV Año (Dimensión 4).

\section{Percepción sobre la Motivación para Emprender}

La escala para medir la percepción sobre la Motivación de los Estudiantes para Emprender consta de cinco afirmaciones, que a continuación se analizarán. Los estudiantes de I Año obtienen mayor grado de certeza respecto a la motivación para emprender, particularmente en los ítems 3.2, 3.3 y 3.4.

Tabla 8.

Motivación para emprender

\begin{tabular}{cccc}
\hline Ítem & Grupo de estudio & Incapaz & Capaz \\
\hline 3.1 & I año & $54 \%$ & $46 \%$ \\
& IV año & $43 \%$ & $57 \%$ \\
3.2. & I año & $8 \%$ & $92 \%$ \\
& IV año & $13 \%$ & $87 \%$ \\
3.3. & I año & $25 \%$ & $75 \%$ \\
3.4. & IV año & $39 \%$ & $61 \%$ \\
3.5. & I año & $25 \%$ & $75 \%$ \\
& IV año & $52 \%$ & $48 \%$ \\
& I año & $13 \%$ & $87 \%$ \\
Promedio & IV año & $13 \%$ & $87 \%$ \\
& I año & $\mathbf{2 5 \%}$ & $\mathbf{7 5 \%}$ \\
& IV año & $\mathbf{3 2 \%}$ & $\mathbf{6 8 \%}$ \\
\hline
\end{tabular}

En contraste, los de IV Año tienen mayor grado de certeza respecto a la afirmación de que la creación de nuevas empresas está considerada como una manera adecuada para hacerse rico (3.1). 
En promedio, los estudiantes de I Año poseen mayor certeza $(75 \%)$ en cuanto a la Motivación para Aprender que los de IV Año (68\%), resultando una diferencia de 7 puntos porcentuales.

\section{$\underline{\text { Percepción sobre las normas sociales y culturales }}$}

La escala para medir la percepción sobre las normas sociales y culturales de los estudiantes respecto al emprendimiento consta de siete afirmaciones, cuyos resultados se presentan a continuación.

Tabla 9.

\begin{tabular}{cccc}
\multicolumn{4}{c}{ Percepción sobre las normas sociales y culturales } \\
\hline Ítem & Grupo de estudio & Incapaz & Capaz \\
\hline 4.1 & I año & $17 \%$ & $83 \%$ \\
4 & IV año & $35 \%$ & $65 \%$ \\
4.2. & I año & $21 \%$ & $79 \%$ \\
4 & IV año & $26 \%$ & $74 \%$ \\
4.3. & I año & $29 \%$ & $71 \%$ \\
4 & IV año & $43 \%$ & $57 \%$ \\
4.4. & I año & $12 \%$ & $88 \%$ \\
4.5. & IV año & $39 \%$ & $61 \%$ \\
4 & I año & $33 \%$ & $67 \%$ \\
4.6. & IV año & $39 \%$ & $61 \%$ \\
4 & I año & $13 \%$ & $87 \%$ \\
4.7. & IV año & $30 \%$ & $70 \%$ \\
& I año & $21 \%$ & $79 \%$ \\
Promedio & IV año & $35 \%$ & $65 \%$ \\
\hline & I año & $\mathbf{2 1 \%} \%$ & $\mathbf{7 9} \%$ \\
& IV año & $\mathbf{3 5 \%}$ & $\mathbf{6 5 \%}$ \\
\hline
\end{tabular}

Los estudiantes de I Año tienen mayor grado de certeza respecto a la percepción sobre las normas sociales y culturales respecto al emprendimiento, en promedio con una diferencia de catorce puntos porcentuales.

Particularmente, la mayor diferencia la encontramos de 27 puntos porcentuales en lo que respecta a la aseveración 4.4, por lo que los alumnos de I Año piensan que la sociedad estimula la asunción del riesgo empresarial, mientras que los de IV Año no consideran que esto sea tan cierto.

\section{Percepción de Oportunidades}

La escala para medir la percepción sobre las oportunidades para emprender consta de siete afirmaciones.

Los estudiantes de I Año obtienen mayor grado de certeza respecto a la Percepción de Oportunidades para emprender, en casi todos los ítems evaluados, con excepción de lo relacionado con el ítem 5.4, que corresponde a la percepción de que las buenas oportunidades para la creación de nuevas empresas han aumentado considerablemente en los últimos cinco años. 
Invest. pens. crit.

Vol. 3, No. 3, septiembre-diciembre 2015

pp. $13-26$

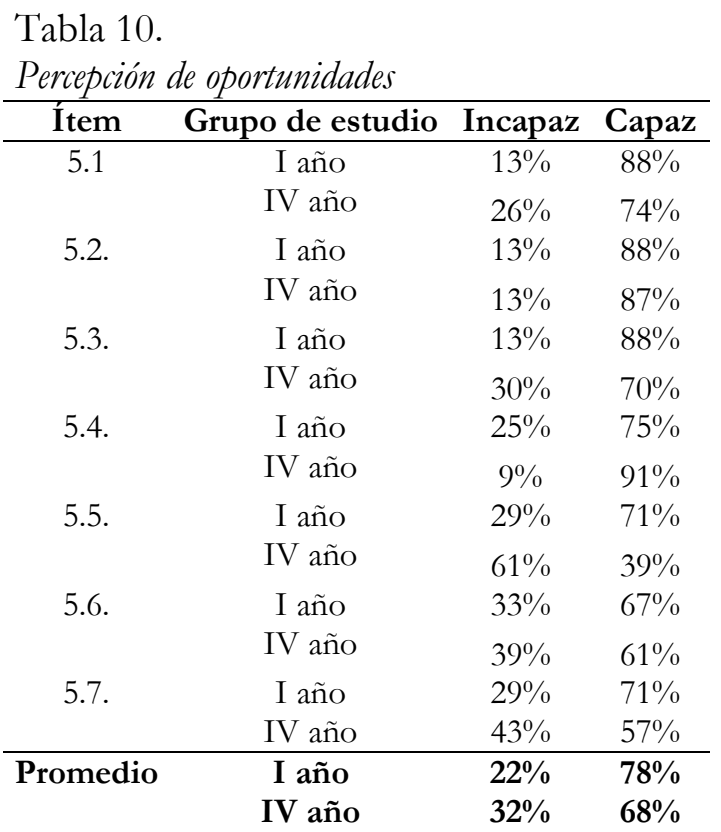

En especial, la mayor diferencia (32 puntos porcentuales) se da en el ítem 5.5, donde casi 3 de cada 4 (71\%) estudiantes de I Año consideran que es fácil dedicarse a explotar oportunidades empresariales, mientras que aproximadamente solo 2 de cada 5 (39\%) de IV Año piensan esto.

\section{Discusión}

La presente investigación ha perseguido evaluar la hipótesis de que existen diferencias significativas en la intención emprendedora y la actitud hacia el emprendimiento en dos grupos de estudiantes de Ingeniería Industrial Administrativa de la USMA: alumnos de Primer Ingreso (recién iniciando su carrera universitaria) y estudiantes de último año de la carrera (graduandos), teniendo el grupo graduando mayores niveles de autoeficacia así como actitud hacia el emprendimiento.

Los resultados obtenidos respaldan el hecho de que los jóvenes graduandos tienen mayor nivel de autoeficacia, entendida como la capacidad para crear y gestionar una nueva empresa con éxito. Sin embargo, respecto hacia la actitud hacia el emprendimiento, los resultados señalan que los de Primer Ingreso tienen una mejor actitud.

Una posible explicación de ambos hallazgos, es entendible para el grupo de graduandos, dado que la creencia de mayores niveles de autoeficacia es compatible con la etapa de formación que estos viven (último año de estudios y próximos a elegir una opción de carrera), han sido preparados debidamente para crear y/o gestionar una empresa con éxito, en contraposición con los que empiezan a adquirir los conocimientos, habilidades y destrezas para operar en el mercado. 
En relación con los resultados mostrados por los estudiantes de Primer Ingreso respecto a las actitudes hacia el emprendimiento, en comparación con los graduandos, existe la percepción que actualmente los más jóvenes tienen mayores deseos de emprender (Department of Labor of the United States of America, 2008, citado en Montes, s.f.), como una manera de actuar y pensar en la vida. Existe mayor reconocimiento a cuestiones tales como el refuerzo de la autonomía e iniciativa personal, la asunción de riesgo empresarial, el estímulo hacia la creatividad y la innovación, se perciben muchas y buenas oportunidades para la creación de nuevas empresas y una mejor percepción hacia el emprendedor como una figura que goza de gran reconocimiento y prestigio social.

En términos de País, además, se puede explicar por el elevado crecimiento económico mostrado durante los últimos 5 años, situando nuestro PIB en 8.5\%, promedio anual (Banco Mundial, 2015), lo que nos coloca como una de las economías más dinámicas en el mundo y, además, con un elevado espacio para oportunidades de negocio.

Si añadimos al hecho que es durante los últimos 5 años que se ha dado una explosión del fenómeno emprendedor, con el desarrollo de un ecosistema que está mejorando el entorno amigable hacia el emprendedor, con acciones desde transformación de proyectos de vida hacia el emprendimiento hasta el apoyo técnico y financiero para la identificación de ideas de negocios, formulación, puesta en marcha y aceleración, con diversos y variados eventos celebrados anualmente desde la academia, el sector público y privado, y en espacios creados por los propios emprendedores (comunidad emprendedora), nos indica que son espacios que no existían hace 5 años.

Hoy día todo el esfuerzo está confluyendo en una Mesa Técnica Nacional de Emprendimiento que cada vez más muestra trabajo colaborativo y en redes, entre instituciones e individuos, lo cual es percibido por los más jóvenes.

Hemos experimentado desde cambios culturales, procesos incipientes aún en el sistema educativo, mayores condiciones de la demanda relacionadas a motores de crecimiento, nuevos programas de ciencia tecnología e innovación, mayores posibilidades de financiamiento para crear y hacer crecer los emprendimientos (desde etapa temprana hasta en etapa de expansión), mayor capital social que permite tejer puentes para desarrollar redes de contacto y, por último, cambios en políticas y regulaciones que han resultado más amigables a los emprendedores.

Todos estos factores ambientales y sistémicos son aprovechados por los más jóvenes, quienes, en síntesis, perciben una mayor apertura de mentalidad y cultura hacia el emprendimiento en el país y, por ende, tienen una mejor actitud hacia el mismo.

\section{Referencias}

Banco Mundial (2015). Panamá. Disponible en Banco Mundial http://www.bancomundial.org/es/country/panama 
Invest. pens. crit.

Vol. 3, No. 3, septiembre-diciembre 2015

pp. $13-26$

Bandura, A. (1977). Self-efficacy toward a unifying theory of behavior change. Psychological Review, 84, 191-215.

De Noble, A. F., Dong, J. y Sanford, B. E. (1999). Entrepreneurial self-efficacy: The development of a measure and its relationship to entrepreneurial action. En R.D. Reynolds, W.D. Bygrave, S. Manigart, C.M. Mason, G.D. Meyer, H.J. Sapienza y K.G. Shaver (eds) Frontiers for Entrepreneurship Research. Massachusetts , EEUU: P\&R Publication, pp.73-87.

Elfving, J., Brännback, M. y Carsud, A. (2009). Toward a contextual model of entrepreneurial intentions. En A. Carsud y M. Brännback (eds.) Understanding the entrepreneurial mind (pp. 23-33). Nueva York, EEUU: Springer.

Fundación Ciudad del Saber [FCS], y Fundación de Estudios Avanzados de Gerencia [IESA]. (2009). Global entrepreneurship monitor: Panamá 2009. Panamá, Panamá: Autor.

Fundación Ciudad del Saber [FCS], y Fundación de Estudios Avanzados de Gerencia [IESA]. (2011). Global entrepreneurship monitor: Panamá 2011. Panamá, Panamá: Autor.

Montes, C. (2012). Promoviendo la capacidad de innovación de la mipyme. Conferencia presentada en el II Diálogo Interamericano de Altas Autoridades de MIPYMEs, Nueva Orleans, EEUU.. Disponible en: http://www.oas.org/en/sedi/desd/IIDialogo/presentations/Camilo_Montes.pdf

Moriano, J.A., Palací, F. J. y Morales, J. F. (2006). Adaptación y validación en España de la escala de Autoeficacia Emprendedora. Revista de Psicología Social, 21, 51-64.

Sánchez, J. C. (2015). La figura del emprendedor: Sensibilización y fomento. Salamanca, España: Universidad de Salamanca.

Shapero, A. y Sokol, L. (1982). The social dimensions of entrepreneurship. En C. Kent, D. Sexton y K. Vesper (eds.) Encyclopedia of Entrepreneurship (72-90). 